\title{
Optimal Design of Nonuniform FIR Transmultiplexer Using Semi-infinite Programming
}

\author{
Charlotte Yuk-Fan Ho, Member, IEEE, Bingo Wing-Kuen Ling, Yan-Qun Liu, Peter Kwong-Shun Tam, \\ Member, IEEE, Kok-Lay Teo, Senior Member, IEEE
}

\begin{abstract}
This paper considers an optimum nonuniform FIR transmultiplexer design problem subject to specifications in the frequency domain. Our objective is to minimize the sum of the ripple energy for all the individual filters, subject to the specifications on amplitude and aliasing distortions, and to the passband and stopband specifications for the individual filters. This optimum nonuniform transmultiplexer design problem can be formulated as a quadratic semi-infinite programming problem. The dual parametrization algorithm is extended to this nonuniform transmultiplexer design problem. If the lengths of the filters are sufficiently long and the set of decimation integers is compatible, then a solution exists. Since the problem is formulated as a convex problem, if a solution exists, then the solution obtained is unique and the local solution is a global minimum.
\end{abstract}

Index Terms - Nonuniform transmultiplexer, semi-infinite programming, dual parameterization.

\section{INTRODUCTION}

$\mathrm{T}$ RANSMULTIPLEXERS are important in multimedia signal processing and communication systems because several data signals can be transmitted through a single channel by using time division multiplexing or frequency division multiplexing techniques [1]. The design of uniform transmultiplexers can be found in [1]. Since the design of uniform transmultiplexers can be based on the design of uniform filter banks, the existing theory for the design of uniform filter banks are adequate and there are many design

Manuscript received February 6, 2004; revised May 3, 2004. This work was substantially supported by a research grant (project number G-YD26) from the Hong Kong Polytechnic University, the Centre for Multimedia Signal Processing, The Hong Kong Polytechnic University, the CRGC grant (project number PolyU 5101\01E) from the Research Grants Council of Hong Kong, as well as a research grant from Australian Research Council. The associate editor coordinating the review of this manuscript and approving it for publication was Dr. Henrique Malvar.

Charlotte Yuk-Fan Ho is with the Department of Electronic Engineering, Queen Mary, University of London, Bingo Wing-Kuen Ling is with the Department of Electronic Engineering, King's College London, Yan-Qun Liu is with the Department of Mathematics and Statistics, Royal Melbourne Institute of Technology, Peter Kong-Shun Tam is with the Department of Electronic and Information Engineering, and Kok-Lay Teo is with the Department of Applied Mathematics and Centre for Multimedia Signal Processing, Department of Electronic and Information Engineering. (e-mail: wing-kuen.ling@kcl.ac.uk).

Publisher Item Identifier methods available for uniform filter banks. So there is no difficulty on the design of uniform transmultiplexers and the existing design methods work very well in many applications.

Suppose we want to have different bandwidths allocated for different applications and these signals are multiplexed together and transmitted in a single channel. For example, a broader bandwidth is required for the transmission of videos, while a narrower bandwidth is enough for transmitting texts. For another example, it may be necessary to allocate more resources to the more critical transmission applications, such as those for the military and medical purposes, and fewer resources to the less critical applications, such as those for the entertainment. For a further example, resources of the bandwidth may be allocated according to the rates that the consumers can afford. All of these circumstances can be solved by applying a nonuniform transmultiplexer. Similarly, the design of nonuniform transmultiplexers can be based on the design of nonuniform filter banks [2]. However, when the set of decimation integers is incompatible, there will be only one nonzero element in at least one row of the aliasing matrix of the corresponding filter bank system. This structural dependency and the incompatibility cannot be eliminated by using a set of linear time-invariant (LTI) filters and a set of simple samplers [3]. Hence, nonuniform transmultiplexers suffer from amplitude, phase, aliasing and crosstalk distortions, and perfect reconstruction cannot be achieved [3].

Some researchers formulate the nonuniform transmultiplexer design problem as an unconstrained optimization problem so that the transmultiplexer is as close as possible to an exact perfect reconstruction system [2]. Although the unconstrained optimization problem can be solved easily, constraints are often necessary to be imposed for real practical situations. For example, as noise is often presented in the transmultiplexer, filters with good frequency selectivity are required and the passband and stopband specifications for the individual filters are commonly imposed. Besides formulating the perfect reconstruction error as a cost function, another common way is to formulate it as constraints. This is because the cost function may not be convex if the perfect reconstruction error is formulated as cost. Moreover, amplitude and aliasing distortions may be particularly serious at certain 
frequency bands, instead of evenly distributed over the whole frequency spectrum. Hence, by formulating the perfect reconstruction distortion as constraints, excessive amplitude and aliasing distortions at certain frequency bands can be avoided.

Since all the specifications are expressed as continuous constraints in the frequency domain, this optimization problem is actually a semi-infinite programming problem. To tackle a semi-infinite programming problem, one way is to approximate it as a finite dimensional problem through a discretization of the index set of the continuous constraints [6]. Many existing optimization-based design techniques for filters as well as filter banks deal with discretized constraints because the difference between the exact upper bound of a discretized constraint function and that of the corresponding continuous constraint function vanishes as the number of grid points increases. Moreover, since the discretized version of the problem is a standard convex quadratic programming problem, there exists some reliable optimization solvers for solving such quadratic programming problems and those existing solvers allow the use of several thousand grid points. Typically, when the number of grid points is in the range of 1000-2000, the solution obtained for designing a single filter has practically no difference to the globally optimized solution for the corresponding continuous optimization problem. However, for a filter bank design problem, since we need to design more than one filter, the number of grid points involved is more than that for a single filter design problem. The number of grid points for amplitude and aliasing distortions are also usually more than that for the specifications on the passband and stopband of the filters because the polynomial orders of the polynomials for the amplitude and aliasing distortions are much higher than that for the specifications on the passband and stopband of the filters. As a result, the number of grid points required in the problem is usually in the order of magnitude of 10000 . In this situation, the implementation cost using the discretization method is very high and the time required for solving the quadratic programming problem is very long. Furthermore, since the exact minimum number of grid points employed and their exact grid positions are not known, and the minimum number of grid points usually corresponds to nonuniform grid positions, which there are no analytical results discussed on this issue. Hence, more than the minimum number of grid points is usually employed. As a result, some of the computation effort is wasted and this method is not efficient in this sense.

In order to avoid the above difficulties, we propose to solve the problem via the dual parameterization approach, which was developed recently in [4] and [5]. This algorithm is proved to be very efficient and effective. In this paper, we apply this technique to solve an optimum nonuniform transmultiplexer design problem so that the sum of the ripple energy for all the individual filters are minimized, subject to the specifications on the amplitude and aliasing distortions and to the passband and stopband specifications for the individual filters.

This paper is organized as follows. In Section II, we give the notations and definitions that appear throughout this paper. In Section III, we formulate the nonuniform transmultiplexer design problem as a quadratic semi-infinite programming problem. In Section IV, a design example is presented. Finally, we summarize and conclude our works in Section V.

\section{Notation AND Definitions}

Let $x_{i}[n]$ and $y_{i}[n]$, for $i=0,1, \cdots, N-1$, be, respectively, the input and output signals of the nonuniform transmultiplexer shown in Figure 1. Let $\downarrow n_{i}$ and $\uparrow n_{i}$, for $i=0,1, \cdots, N-1$, denote, respectively, the downsamplers and upsamplers, and let $H_{i}(z)$ and $F_{i}(z)$, for $i=0,1, \cdots, N-1$, be, respectively, the filters in the receiver and transmitter. The nonuniform transmultiplexer is said to achieve perfect reconstruction if $y_{i}[n]$ is a delayed gained version of $x_{i}[n]$, for $i=0,1, \cdots, N-1$, that is, $\exists c_{i} \in \Re$ and $\exists m_{i} \in Z$ such that $y_{i}[n]=c_{i} x_{i}\left[n-m_{i}\right]$, for $i=0,1, \cdots, N-1$, where $\Re$ and $Z$ denotes, respectively, the set of real numbers and integers.

\section{PROBLEM FORMULATION}

To avoid phase distortions, either symmetric or antisymmetric even-tap filters are designed, and the filters in the receiver are expressed as:

$$
H_{i}(\omega)=2 e^{-j\left(\omega D_{i}+\phi_{i}\right)} \sum_{k=0}^{N_{i}-1} a_{i, N_{i}-1-k} \cos \left(\left(k+\frac{1}{2}\right) \omega+\phi_{i}\right),
$$

for $i=0,1, \cdots, N-1$, where $D_{i}, \phi_{i}$ and $2 N_{i}$, are respectively, the group delay, phase shift and order of the filter $H_{i}(\omega)$. $\phi_{i}=0$ and $\phi_{i}=\frac{\pi}{2}$ correspond, respectively, to the symmetric and antisymmtric cases. Define $B_{\Omega}=[-\pi, \pi]$, and let the passband and stopbands of $H_{i}(\omega)$ be, respectively, denoted by $B_{i}^{p}$ and $B_{i}^{s}$, for $i=0,1, \cdots, N-1$. The coefficients $a_{i, k} \in \mathfrak{R}$ are to be designed. Let

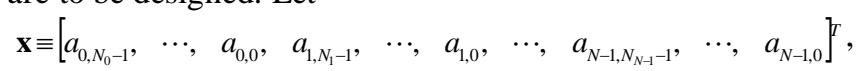
where the superscript ${ }^{T}$ denotes the transpose. Define

$$
\xi_{i}(\omega) \equiv e^{-j\left(\omega D_{i}+\phi_{i}\right)} \boldsymbol{\eta}_{i}(\omega),
$$

where

$$
\boldsymbol{\eta}_{i}(\omega) \equiv 2\left[\cos \left(\frac{1}{2} \omega+\phi_{i}\right), \quad \cos \left(\frac{3}{2} \omega+\phi_{i}\right), \cdots, \quad \cos \left(\frac{2 N_{i}-1}{2} \omega+\phi_{i}\right)\right]^{T},
$$

for $i=0,1, \cdots, N-1$. Then we have:

where

$$
\mathbf{H}(\omega)=\boldsymbol{\xi}(\omega) \mathbf{x},
$$

and

$$
\mathbf{H}(\omega)=\left[H_{0}(\omega), \quad \cdots, \quad H_{N-1}(\omega)\right]^{T}
$$

$$
\xi(\omega)=\operatorname{diag}\left(\left(\xi_{0}(\omega)\right)^{T}, \quad \cdots, \quad\left(\xi_{N-1}(\omega)\right)^{T}\right) .
$$

Assume the desired frequency responses are: 


$$
H_{d_{i}}(\omega)=A_{d_{i}}(\omega) e^{-j \theta_{d_{i}}(\omega)} \text {, for } i=0,1, \cdots, N-1,
$$

where $A_{d_{i}}(\omega)$ and $\theta_{d_{i}}(\omega)$ are real functions representing the desired amplitude and phase responses. The cost reflecting the sum of the ripple energy for all individual filters in the passband and stopband of the filters can be expressed as:

$$
J(\mathbf{x}) \equiv \sum_{i=0}^{N-1} \int_{B_{i}^{p} \cup B_{i}^{s}}\left(\kappa_{i}(\omega)\right)^{2} d \omega,
$$

where

in which

$$
\boldsymbol{\kappa}(\omega)=\boldsymbol{\eta}(\omega) \mathbf{x}-\mathbf{A}_{\mathbf{d}}(\omega),
$$

$$
\begin{gathered}
\boldsymbol{\kappa}(\omega)=\left[\begin{array}{lll}
\kappa_{0}(\omega), & \cdots, & \kappa_{N-1}(\omega)
\end{array}\right]^{T}, \\
\boldsymbol{\eta}(\omega)=\operatorname{diag}\left(\left(\boldsymbol{\eta}_{0}(\omega)\right)^{T}, \quad \cdots, \quad\left(\boldsymbol{\eta}_{N-1}(\omega)\right)^{T}\right),
\end{gathered}
$$

and

$$
\mathbf{A}_{\mathbf{d}}(\omega)=\left[A_{d_{0}}(\omega), \quad \cdots, \quad A_{d_{N-1}}(\omega)\right]^{T} .
$$

It can be shown easily that there exist a positive definite matrix $\mathbf{Q}$, a vector $\mathbf{b}$ and a constant $p$ such that

$$
J(\mathbf{x})=\frac{1}{2} \mathbf{x}^{T} \mathbf{Q} \mathbf{x}+\mathbf{b}^{T} \mathbf{x}+p \cdot
$$

Next, we consider the constraints to be imposed. Let the bounds on the passband and stopband ripples of $H_{i}(\omega)$ be, respectively, $\delta_{p_{i}}$ and $\delta_{s_{i}}$, for $i=0,1, \cdots, N-1$. Then the constraints on the passband and stopband ripples can be expressed as:

and

$$
\left|\kappa_{i}(\omega)\right| \leq \delta_{p_{i}}, \text { for } \omega \in B_{i}^{p} \text { and for } i=0,1, \cdots, N-1,
$$$$
\left|\kappa_{i}(\omega)\right| \leq \delta_{s_{i}}, \text { for } \omega \in B_{i}^{s} \text { and for } i=0,1, \cdots, N-1 \text {. }
$$

It can be shown easily that the constraints can be expressed as follows:

$$
\mathbf{A}_{f}(\omega) \mathbf{x} \leq \mathbf{c}_{f}(\omega) \text {, for } \omega \in B_{0}^{p},
$$

for appropriate $\mathbf{A}_{f}(\omega)$ and $\mathbf{c}_{f}(\omega)$.

In order to capture the specifications on the amplitude and aliasing distortions in the design, we swap the transmitter and receiver and obtain a nonuniform filter bank system. If the corresponding nonuniform filter bank system achieves perfect reconstruction with the delay of the whole system equal to an integer multiple of $N$, then the corresponding transmultiplexer achieves perfect reconstruction. Hence, in this paper, we formulate the perfect reconstruction problem of the nonuniform transmultiplexer as that of the corresponding nonuniform filter bank. For the above situation, the corresponding nonuniform transmultiplexer can achieve perfect reconstruction. Although the perfect reconstruction condition of a general nonuniform transmultiplexer is not the same as that of the corresponding nonuniform filter bank, and actually, we over-constrain the transmultiplexer design problem, this problem formulation can simplify the problem.

Let

$$
F_{i}(\omega)=2 e^{-j\left(\omega D_{i}^{\prime}+\phi_{i}^{\prime}\right.} \sum_{k=0}^{N_{i}^{\prime}-1} b_{i, N_{i}-1-k} \cos \left(\left(k+\frac{1}{2}\right) \omega+\phi_{i}^{\prime}\right),
$$

for $i=0,1, \cdots, N-1$, where $D_{i}^{\prime}, \quad \phi_{i}^{\prime}$ and $2 N_{i}^{\prime}$, are respectively, the group delay, phase shift and order of the filter $F_{i}(\omega)$ with the coefficients $b_{i, k} \in \mathfrak{R}$. Define

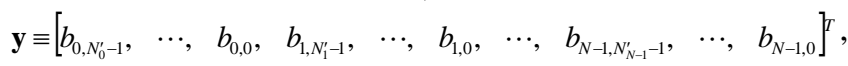
then

where

$$
\mathbf{F}(\omega)=\xi^{\prime}(\omega) \mathbf{y}
$$

and

$$
\mathbf{F}(\omega)=\left[\begin{array}{lll}
F_{0}(\omega), & \cdots, \quad F_{N-1}(\omega)
\end{array}\right]^{T}
$$

in which

$$
\xi^{\prime}(\omega)=\operatorname{diag}\left(\left(\xi_{0}^{\prime}(\omega)\right)^{T}, \quad \cdots, \quad\left(\xi_{N-1}^{\prime}(\omega)\right)^{T}\right),
$$

$$
\xi_{i}^{\prime}(\omega) \equiv e^{-j\left(\omega D_{i}^{\prime}+\phi_{i}^{\prime}\right)} \boldsymbol{\eta}_{i}^{\prime}(\omega)
$$

and

$$
\boldsymbol{\eta}_{i}^{\prime}(\omega) \equiv 2\left[\cos \left(\frac{1}{2} \omega+\phi_{i}^{\prime}\right), \quad \cos \left(\frac{3}{2} \omega+\phi_{i}^{\prime}\right), \quad \cdots, \quad \cos \left(\frac{2 N_{i}^{\prime}+1}{2} \omega+\phi_{i}^{\prime}\right)\right]^{T},
$$

for $i=0,1, \cdots, N-1$. Let $M$ be the least common multiple of the decimation integers. Define $k_{i}=\frac{M}{n_{i}}$ and

$$
\mathbf{v}_{i, j}(\omega) \equiv \frac{1}{n_{i}}\left[H_{i}\left(\omega-\frac{2 \pi j}{n_{i}}\right), \quad 0, \quad \cdots, \quad 0\right],
$$

for $i=0,1, \cdots, N-1$, and for $j=0,1, \cdots, n_{i}-1$. There are $k_{i}-1$ zeros in the vector $\mathbf{v}_{i, j}(\omega)$. Let

$$
\mathbf{v}_{i}(\omega) \equiv\left[\mathbf{v}_{i, 0}(\omega), \quad \cdots, \quad \mathbf{v}_{i, n_{i}-1}(\omega)\right]^{T}
$$

for $i=0,1, \cdots, N-1$. Then, the aliasing matrix of the corresponding nonuniform filter bank system is:

$$
\mathbf{V}(\omega)=\left[\mathbf{v}_{0}(\omega), \quad \boldsymbol{v}_{1}(\omega), \quad \cdots, \quad \boldsymbol{v}_{N-1}(\omega)\right]
$$

Let the input of the corresponding nonuniform filter bank system be $X(\omega)$ and define

$$
\mathbf{X}_{l}(\omega) \equiv\left[X(\omega), \quad X\left(\omega-\frac{2 \pi}{M}\right), \quad \cdots, \quad X\left(\omega-\frac{2 \pi(M-1)}{M}\right)\right],
$$

then

$$
Y(\omega)=\mathbf{X}_{l}(\omega) \mathbf{V}(\omega) \mathbf{F}(\omega),
$$

where $Y(\omega)$ is the output of the corresponding nonuniform filter bank system and

$$
\mathbf{F}(\omega) \equiv\left[F_{0}(\omega), \quad F_{1}(\omega), \quad \cdots, \quad F_{N-1}(\omega)\right]^{T} .
$$

Define

$$
\boldsymbol{\mu}(\omega) \equiv\left[\begin{array}{llll}
c e^{-j m \omega}, & 0, & \cdots, & 0
\end{array}\right]^{T},
$$

where $c$ and $m$ are, respectively, the gain and the delay of the corresponding nonuniform filter bank system. Let

$$
\mathbf{E}(\omega) \equiv|\mathbf{V}(\omega) \mathbf{F}(\omega)-\boldsymbol{\mu}(\omega)|,
$$

where $|\cdot|$ denotes the absolute values of the elements in the vector $\mathbf{V}(\omega) \mathbf{F}(\omega)-\boldsymbol{\mu}(\omega)$. Define $E_{i}(\omega)$ be the $(i+1)^{t h}$ element of the vector $\mathbf{E}(\omega)$. Then, the amplitude distortion of the corresponding nonuniform filter bank system is reflected by $E_{0}(\omega)$ and the aliasing distortion is reflected by $E_{i}(\omega)$ for $i=1,2, \cdots, M-1$.

Let the bounds on the amplitude and aliasing distortions be, respectively, $\delta_{a}$ and $\delta_{A}$. Then the constraints on the 
amplitude and aliasing distortions of the corresponding nonuniform filter bank system can be expressed as:

$$
E_{0}(\omega)=\left|\mathbf{y}^{T} \mathbf{Q}_{0}(\omega) \mathbf{x}-c e^{-j m \omega}\right| \leq \delta_{a}, \text { for } \omega \in B_{\Omega},
$$

and

$$
\begin{gathered}
E_{i}(\omega)=\left|\mathbf{y}^{T} \mathbf{Q}_{i}(\omega) \mathbf{x}\right| \leq \delta_{A}, \text { for } \omega \in B_{\Omega} \text { and for } \\
i=1,2, \cdots, M-1,
\end{gathered}
$$

where

$\mathbf{Q}_{i}(\omega)=\operatorname{diag}\left(\mathbf{Q}_{0}^{i}(\omega), \quad \cdots, \quad \mathbf{Q}_{N-1}^{i}(\omega)\right)$, for $i=0,1, \cdots, M-1$, in which

$$
\mathbf{Q}_{j}^{i}(\omega)=\left\{\begin{array}{cc}
\frac{1}{n_{j}} \xi_{j}^{\prime}(\omega)\left(\xi_{j}\left(\omega-\frac{2 \pi i}{M}\right)\right)^{T} & \frac{i}{k_{j}} \in Z \\
\mathbf{0} & \text { otherwise }
\end{array} .\right.
$$

Since $\mathbf{Q}_{i}(\omega)$ and $c e^{-j m \omega}$ are complex functions, the constraints taking the absolute value of complex functions are quadratic with respect to $\mathbf{x}$. One common way to tackle this kind of problem is via discretization. If the discretization approach is applied, then the constraints become convex quadratic constraints which will lead to a quadratic programming problem with convex quadratic constraints. Problems of this type belong to the class of convex programming problems and admit a unique global solution if a solution exists. There are some available public domain convex programming solvers for solving this type of quadratic programming problems. However, as mentioned in the introduction section, the number of grid points is very large and the computation complexity is also very high, so we approximate the quadratic constraints as linear constraints as described below.

Consider the following constraints:

$$
\begin{aligned}
& \left|\operatorname{Re}\left\{\mathbf{y}^{T} \mathbf{Q}_{0}(\omega) \mathbf{x}-c e^{-j m \omega}\right\}\right| \leq \delta_{a}^{R}, \text { for } \omega \in B_{\Omega}, \\
& \left|\operatorname{Im}\left\{\mathbf{y}^{T} \mathbf{Q}_{0}(\omega) \mathbf{x}-c e^{-j m \omega}\right\}\right| \leq \delta_{a}^{I}, \text { for } \omega \in B_{\Omega},
\end{aligned}
$$

$\left|\operatorname{Re}\left\{\mathbf{y}^{T} \mathbf{Q}_{i}(\omega) \mathbf{x}\right\}\right| \leq \delta_{A}^{R}$, for $\omega \in B_{\Omega}$ and for $i=1,2, \cdots, M-1$, and

$\left|\operatorname{Im}\left\{\mathbf{y}^{T} \mathbf{Q}_{i}(\omega) \mathbf{x}\right\}\right| \leq \delta_{A}^{I}$, for $\omega \in B_{\Omega}$ and for $i=1,2, \cdots, M-1$, where $\operatorname{Re}\{\cdot\}$ and $\operatorname{Im}\{\cdot\}$ denote, respectively, the real and imaginary parts of the functions inside the brackets; $\delta_{a}^{R}$ and $\delta_{a}^{I}$ denote, respectively, the constraints on the real and imaginary parts of the amplitude distortion of the corresponding nonuniform filter bank system; $\delta_{A}^{R}$ and $\delta_{A}^{I}$ denote, respectively, the constraints on the real and imaginary parts of the aliasing distortion of the corresponding nonuniform filter bank system.

Note that each absolute value constraint is not equivalent to its corresponding real/imaginary value constraints. The reason for this is that the mapping between these two constraints is not invertible. However, the problem is much simplified and the approximation is valid if $\delta_{a}$ and $\delta_{A}$ are small enough.

If the set of filters in the transmitter is selected a prior, then $\mathbf{y}$ is known. The constraints can be expressed as:

$$
\mathbf{A}_{r}(\omega) \mathbf{x} \leq \mathbf{c}_{r}(\omega) \text {, for } \omega \in B_{0}^{p},
$$

and

$$
\mathbf{A}_{r}(\omega)=\left[\begin{array}{lll}
\left(\mathbf{A}_{r}^{0}(\omega)\right)^{T}, & \cdots, & \left(\mathbf{A}_{r}^{M-1}(\omega)\right)^{T}
\end{array}\right]^{T}
$$

in which for $i=0,1, \cdots, M-1$,

$$
\mathbf{c}_{r}(\omega)=\left[\left(\mathbf{c}_{r}^{0}(\omega)\right)^{T}, \quad \cdots, \quad\left(\mathbf{c}_{r}^{M-1}(\omega)\right)^{T}\right]^{T},
$$

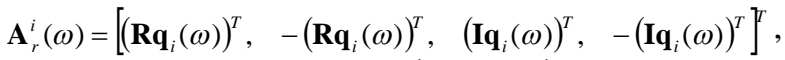
$\mathbf{R q}_{i}(\omega)=\operatorname{Re}\left\{\mathbf{y}^{T} \mathbf{Q}_{i}(\omega)\right\}$$$
\mathbf{I q}_{i}(\omega)=\operatorname{Im}\left\{\mathbf{y}^{T} \mathbf{Q}_{i}(\omega)\right\},
$$

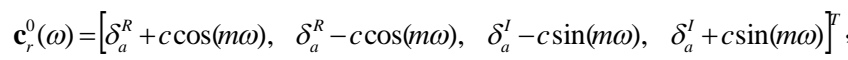
and

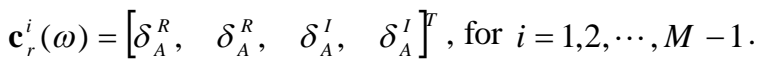

Define

$$
\mathbf{A}(\omega)=\left[\begin{array}{c}
\mathbf{A}_{f}(\omega) \\
\mathbf{A}_{r}(\omega)
\end{array}\right], \text { for } \omega \in B_{0}^{p}
$$

and

$$
\mathbf{c}(\omega)=\left[\begin{array}{l}
\mathbf{c}_{f}(\omega) \\
\mathbf{c}_{r}(\omega)
\end{array}\right], \text { for } \omega \in B_{0}^{p} .
$$

Consequently, the nonuniform transmultiplexer design problem can be formulated as the following semi-infinite programming problem:

$$
\begin{array}{ll}
\min _{\mathbf{x}} & J(\mathbf{x})=\frac{1}{2} \mathbf{x}^{T} \mathbf{Q} \mathbf{x}+\mathbf{b}^{T} \mathbf{x}+p, \\
\text { subject to } & \mathbf{g}(\mathbf{x}, \omega)=\mathbf{A}(\omega) \mathbf{x}-\mathbf{c}(\omega) \leq \mathbf{0}, \text { for } \omega \in B_{0}^{p},
\end{array}
$$

where $\mathbf{A}(\omega)$ and $\mathbf{c}(\omega)$ are continuously differentiable with respect to $\omega \in B_{0}^{p}$.

If the lengths of the filters are sufficiently long, by applying the dual parameterization technique [4], then a solution will exist. Since the problem is formulated as a convex problem, the solution is unique and any local solution is a global solution. Once we obtain the set of filter coefficients in the receiver, we use the same method to compute the filters coefficients in the transmitter, and iterate the above procedures. Since the feasible set of each iteration is convex, this iteration process is actually a projection on a convex set. Hence, the convergence of the iteration process is guaranteed if a solution exists for each iteration. Eventually, a set of transmitter and receiver filters coefficients that globally minimizes the cost function and satisfies the continuous constraints is obtained.

\section{DESIGN EXAMPLE}

Consider a 3-channel nonuniform transmultiplexer with the set of decimation integers $\{2,4,4\}$. Let the transition bandwidths of the lowpass, bandpass and highpass filters be, respectively, $0.3,0.25$ and 0.15 . The real and imaginary parts of both the amplitude and aliasing distortions are bounded by $5 \times 10^{-3}$. The specifications on the passband of the lowpass, bandpass and highpass filters are, respectively, $0.0036,0.0059$ and 0.0033 , and that of the stopband are, respectively, $0.0081,0.0036$ and 0.0081 . The filter length of the lowpass, bandpass and highpass filters are selected as, respectively, $\quad N_{0}=N_{0}^{\prime}=31 \quad, \quad N_{1}=N_{1}^{\prime}=94 \quad$ and

where 
$N_{2}=N_{2}^{\prime}=94$. The parameters for the corresponding nonuniform filter bank are chosen as $m=189$ and $c=1$, while the desired amplitude responses of the filters are defined as $A_{d_{i}}(\omega)=\left\{\begin{array}{ll}1 & \omega \in B_{i}^{p} \\ 0 & \omega \in B_{i}^{s}\end{array}\right.$ for $i=0,1,2$.

Based on the above specifications and the parameters chosen, the nonuniform transmultiplexer design problem can be readily formulated as a semi-infinite programming problem discussed in Section III. It can then be solved via the dual parameterization method. For the details of the theory and the algorithm of solving semi-infinite programming problem via the dual parameterization method, the readers can refer to [4] and [5].

By applying the dual parameterization method, a solution is obtained for a given set of transmitter coefficients. After three iterations between the filters designed for the transmitter and the receiver, the filter coefficients in both the transmitter and receiver converge. The magnitude response of the receiver filters and the transmitter filters are plotted as solid lines in, respectively, Figure 2 and Figure 3. The amplitude and aliasing distortions are shown in Figure 4. For implementing the existing method, we employ 15674 grid points for the discretization method via the convex quadratic programming solver in MATLAB. The magnitude response of the receiver filters and the transmitter filters are plotted as dotted line in, respectively, Figure 2 and Figure 3. The corresponding amplitude and aliasing distortions are shown in Figure 4 . We compared the results and found that the continuous constraints between some discretization points are not satisfied. Our algorithm overwhelmingly produces better results on suppressing the amplitude and aliasing distortions. According to the computer simulation, our algorithm can obtain about $250 \mathrm{~dB}$ improvement for the real part and imaginary part of both the amplitude and aliasing distortions. The amplitude distortion and the aliasing distortion are dramatically reduced from the order of $10^{-3}$ to $10^{-15}$ and $10^{-3}$ to $10^{-18}$, respectively.

To further demonstrate the effectiveness of our proposed algorithm, three random signals with zero mean and unit variance are taken as the input signals for the nonuniform transmultiplexer. The mean square errors of these three channels are $1.3063 \times 10^{-29}, \quad 2.7917 \times 10^{-29}$ and $8.8908 \times 10^{-30}$ respectively. They are negligible for most engineering applications and the nonuniform transmultiplexer can be regarded as a perfect reconstruction system.

\section{CONCLUSION}

The main contribution of this paper is to formulate a nonuniform FIR transmultiplexer design problem as a semi-infinite programming problem. The method is based on the dual parameterization method. One of the advantages of this method is that it is guaranteed to achieve the global minimum while satisfying the passband and stopband specifications as well as the amplitude and aliasing requirements if the solution exists. It also avoids the problems occurred in the existing semi-definite programming approaches [6].

\section{ACKNOWLEDGEMENT}

The work obtained in this paper was supported by a research grant (project number G-YD26) from the Hong Kong Polytechnic University, the Centre for Multimedia Signal Processing, The Hong Kong Polytechnic University, the CRGC grant (project number PolyU 5101\01E) from the Research Grants Council of Hong Kong, as well as a research grant from Australian Research Council.

\section{REFERENCES}

[1] R. D. Koilpillai, T. Q. Nguyen, and P. P. Vaidyanathan, "Some results in the theory of crosstalk-free transmultiplexers," IEEE Transactions on Signal Processing, vol. 39, no. 10, pp. 2174-2183, 1991.

[2] T. Liu, and T. Chen, "Design of multichannel nonuniform transmultiplexers using general building blocks," IEEE Transactions on Signal Processing, vol. 49, no. 1, pp. 91-99, 2001.

[3] P. Q. Hoang, and P. P. Vaidyanathan, "Non-uniform multirate filter banks: theory and design," IEEE International Symposium on Circuits and Systems, ISCAS, vol. 1, pp. 371-374, 1989.

[4] Y. Liu, K.L. Teo and S. Ito, "Global optimization in quadratic semi-infinite programming," Computing, vol. 15, pp. 119-132, 2001.

[5] C. H. Tseng, K. L. Teo, Z. Zang and A. Cantoni, "A dual approach to continuous-time envelope-constrained filter design via orthonormal filters," IEEE Transactions on Circuits and Systems - I: Fundamental Theory and Applications, vol. 46, no. 9, pp. 1042-1054, 1999.

[6] W. S. Lu, "A unified approach for the design of 2-D digital filters via semidefinite programming," IEEE Transactions on Circuits and Systems-I: Fundamental Theory and Applications, vol. 49, no. 6, pp. 814-826, 2002. 

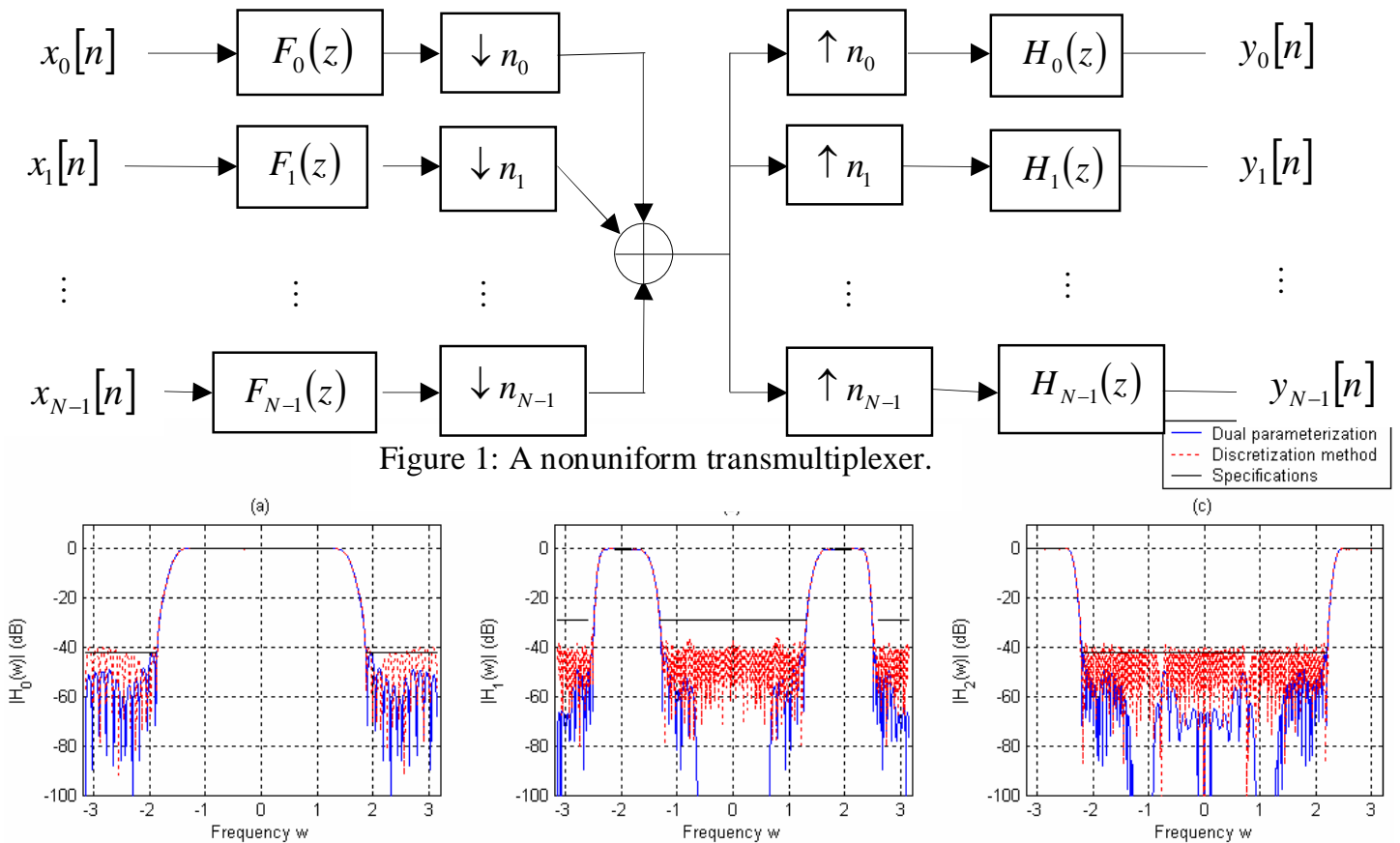

Figure 2: Filter responses of filters in the receiver.
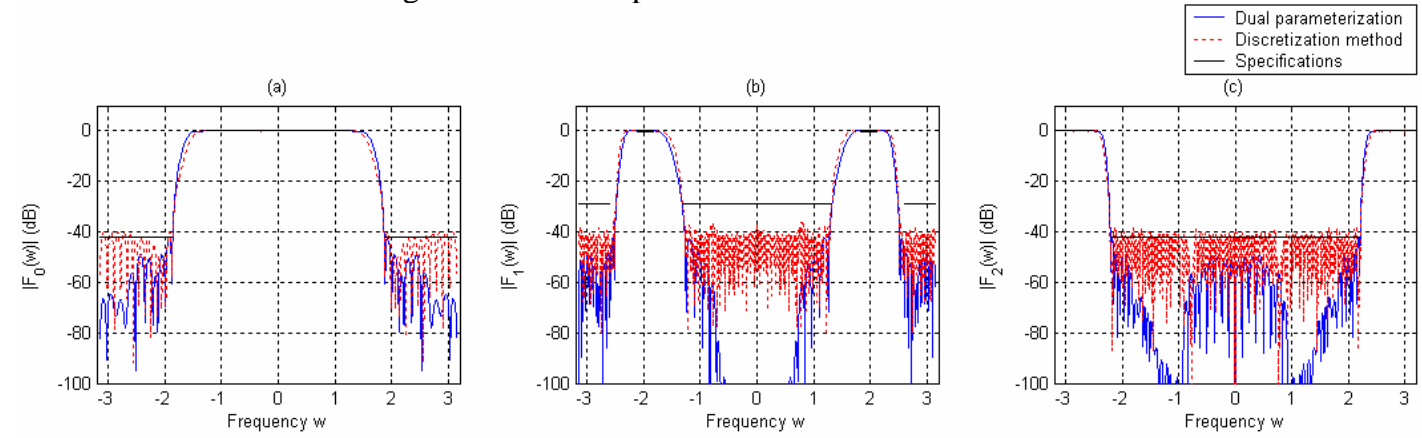

Figure 3: Filter responses of filters in the transmitter.
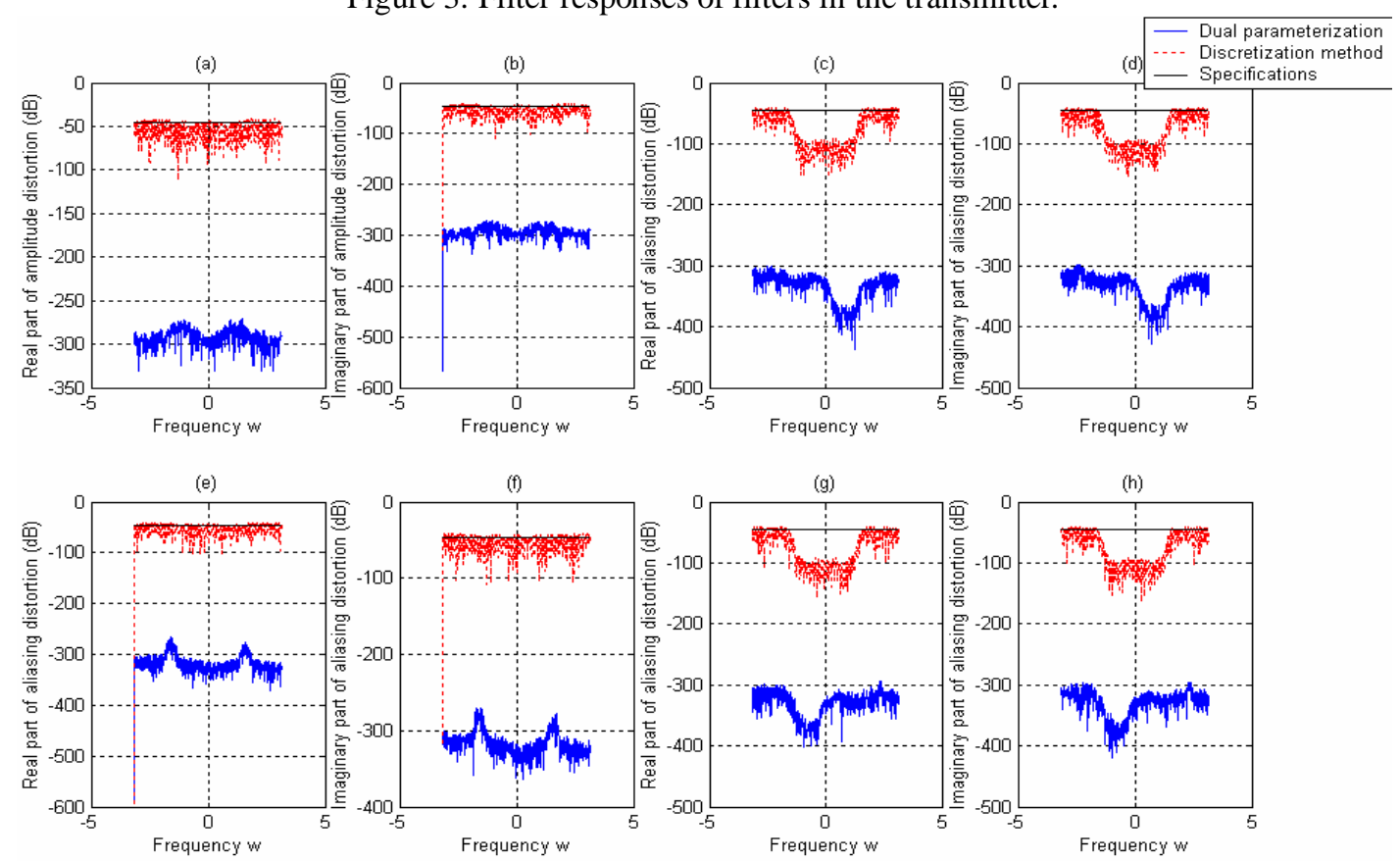

Figure 4: Amplitude and aliasing distortions. 